\title{
The Benefits of Synchronous Collaborative Information Visualization: Evidence from an Experimental Evaluation
}

\begin{abstract}
Sabrina Bresciani and Martin J. Eppler
Abstract-A great corpus of studies reports empirical evidence of how information visualization supports comprehension and analysis of data. The benefits of visualization for synchronous group knowledge work, however, have not been addressed extensively. Anecdotal evidence and use cases illustrate the benefits of synchronous collaborative information visualization, but very few empirical studies have rigorously examined the impact of visualization on group knowledge work. We have consequently designed and conducted an experiment in which we have analyzed the impact of visualization on knowledge sharing in situated work groups. Our experimental study consists of evaluating the performance of 131 subjects (all experienced managers) in groups of 5 (for a total of 26 groups), working together on a real-life knowledge sharing task. We compare (1) the control condition (no visualization provided), with two visualization supports: (2) optimal and (3) suboptimal visualization (based on a previous survey). The facilitator of each group was asked to populate the provided interactive visual template with insights from the group, and to organize the contributions according to the group consensus. We have evaluated the results through both objective and subjective measures. Our statistical analysis clearly shows that interactive visualization has a statistically significant, objective and positive impact on the outcomes of knowledge sharing, but that the subjects seem not to be aware of this. In particular, groups supported by visualization achieved higher productivity, higher quality of outcome and greater knowledge gains. No statistically significant results could be found between an optimal and a suboptimal visualization though (as classified by the pre-experiment survey). Subjects also did not seem to be aware of the benefits that the visualizations provided as no difference between the visualization and the control conditions was found for the self-reported measures of satisfaction and participation. An implication of our study for information visualization applications is to extend them by using real-time group annotation functionalities that aid in the group sense making process of the represented data.
\end{abstract}

Index Terms_Laboratory Studies, Visual Knowledge Representation, Collaborative and Distributed Visualization, synchronous situated collaboration, group work, experiment, knowledge sharing.

\section{INTRODUCTION}

Within the field of information visualization, several researchers have investigated how visualization can support strategic thinking, by reducing cognitive load, offloading short-term memory, allowing for easier comparisons, and generally facilitating inferences [37, 43, 46]. Anecdotal evidence on the usefulness of visualization for supporting explanations and reasoning processes abounds in the literature [44], as well as in popular sayings, as in the famous line "A picture is worth a thousand words": Larkin and Simon [22] have investigated how this saying can be true (and why) in supporting individual reasoning.

Collaborative knowledge work, such as experience sharing and decision making in meetings, is a crucial task in organizations: the quality of the decision process and outcome can have a dramatic impact on a company's performance. Professional consultant have successfully employed visualization in meeting facilitation $[4,16$, 30] and their anecdotal evidence shows that visualization is particularly useful because it helps structuring cognitive processes, for its permanence (as opposed to verbal interventions), and because it support a more focused discussion. The aim of our research is thus to show the relevance and benefits of information visualization for this collaborative, often qualitative group deliberation context. Shneiderman believes that focusing on understanding collaboration is crucial, arguing that "understanding these collaboration-centered

- Sabrina Bresciani is with the University of Lugano, E-mail: sabrina.bresciani@usi.ch.

- Martin J. Eppler is with the University of St. Gallen, E-mail: epplerm@gmail.com.

Manuscript received 31 March 2009; accepted 27 July 2009; posted online 11 October 2009; mailed on 5 October 2009.

For information on obtaining reprints of this article, please send email to: tvcg@computer.org . socio-technical systems could accelerate their adoption and raise their benefits." [36]. Responding to Shneiderman's call to action for rigorous evaluation work in the collaborative visualization field, we have devised an experimental design that is both rigorous and, in terms of the user tasks, close to reality. Among the range of possible evaluation methods $[1,38]$, we have chosen the controlled (betweengroup) experiment as it is considered one of the strongest and most appropriate methods for (summative) system evaluation [42].

The general research question that we aim to answer can be articulated as follows: How does real-time, interactive visualization (or template-based mapping) of participants' contributions affect knowledge sharing in teams? Within this broad question, we focus on synchronous and co-located group work at the managerial level.

Our contribution hence focuses on the effects of visualizing group conversations in real-time (through software and an untrained facilitator) with regard to reasoning and communication purposes, in the context of organizations. We adopt an experimental approach, comparing groups of 5 managers (including a group-assigned facilitator) working together on a knowledge sharing task. Over one hundred managers in 26 groups have participated in one of the three conditions: with visual software support (using fitting or non-fitting templates, as classified through a previous survey among 116 different managers) or with flipchart (control condition). In the treatment conditions the assigned interactive visualization templates are populated by the facilitator with group members' contributions during the knowledge sharing task. The originality of our work lies, we believe, in evaluating the benefits of interactive visualization for group work in an organizational context with a rigorous methodology. This can be relevant to the information visualization community because it uncovers, with solid scientific evidence (given by the experiment methodology and the large number of participants), several advantages of interactive visualization for knowledge sharing. 


\section{RELATED WORK}

This research study aims to apply qualitative, collaborative information visualization to the organizational context. Its roots are mainly in information visualization, human computer interaction [37], and information system studies (in particular in Group Support Systems). It is also motivated by studies on managerial cognition and communication, where decision making processes $[9,45]$ and the impact of group dynamics [25] are described and analyzed.

With regard to the fields of information visualization and human computer interaction, we build on the seminal work of Shneiderman [37], Card et al. [7] and Ware [46] that have examined "the use of computer supported, interactive, visual representations of abstract data to amplify cognition" [7]. More specifically, a few studies have examined the impact of co-located information visualization, such as the work of Suthers [40], Isenberg et al. [21] and Mark et al. [23, 24], although not specifically in the context or organizational or managerial work. Various information visualization scholars have investigated topics similar to the one proposed in our study, such as Chuah et al. [11] who worked on visual knowledge sharing, and Rogers et al. [34] who studied group interactions. The experimental methodology for evaluating information visualization and interactions has been previously used in [10, 32, 33, 47] among others.

In the field of Group Support Systems (GSS) and Computer Supported Cooperative Work (CSCW) there is a long tradition of using experiments, comparing for example, face to face meetings with distributed meetings [3, 4], or comparing different kinds of group supports $[5,8,17]$, or accounting for cross-cultural differences $[26,41]$. These studies, however, have not yet included information visualization evaluations, at best of our knowledge.

Conversely, in the knowledge management and communication community, experiments are not an often used method, with few exceptions [35]. Few, mainly qualitative (and highly cited), studies have examined the effect of boundary objects $[9,39]$ as knowledge transfer and integration devices in organizations. Boundary objects are "objects which are both plastic enough to adapt to local needs and the constraints of the several parties employing them, yet robust enough to maintain a common identity across sites." [39]. Their findings show that, through the use of boundary objects, people from different areas of expertise can bridge their separate knowledge domains, create a shared understanding, and improve decision making.

Finally in the area of management researchers are beginning to explore the potential of using visualization in organizations [16, 35]. These studies typically highlight visualization advantages for different kinds of knowledge work (decision making, knowledge sharing, brainstorming, ranking, etc), mainly through case studybased evidence.

\section{EXPERIMENT}

We have designed and performed an experimental study to assess the impact of visualization for knowledge sharing in co-located groups. Specifically, we have implemented three conditions: groups were assigned either to (1) an optimal (or fitting) visualization support, or (2) to a suboptimal (non-fitting) visualization support, or (3) to a control condition with no visualization support. We have identified the optimal and suboptimal visualizations through a matching questionnaire (see subsection 3.3 for more details). Subjects were randomly divided into groups of five and each group was randomly assigned to one of the conditions, as suitable in most experimental settings [6]. Randomization of subjects is a requirement of experimental settings (in the contrast to quasi-experiments) in order to ensure a non biased distribution of group characteristics.

The task assigned to each group was to share and document knowledge and experiences about business strategy implementation problems in order to come up with a comprehensive list of challenges to consider when implementing a business strategy. This is a highly realistic and relevant management communication task, as many business strategies tend to fail in their implementation stage. The members of the experimental groups were all participants in an executive course on strategic management (see section 3.4) and thus interested in the topic and the task. Each group chose a facilitator who had to capture the members' contributions in front of them, documenting the group's view on the topic. In the two visualization supported condition, the facilitator had to position the contributions (bulleted text items) on the provided interactive graphic templates in a software environment (we have used the en.lets-focus.com package for this task). In the control condition each group was provided with a flipchart and markers. The designated facilitator then wrote down the group members' contributions; the group members were sitting around a table (see Fig. 1) as it is typical in most business meetings.

The two visualization software-supported conditions had the same setting, but used different visualization templates. The group members were sitting around a table and the facilitator was using a laptop connected to a projector, so that all the participants were able to see the results of the visualization of the discussion, projected on a big screen (Fig. 2).

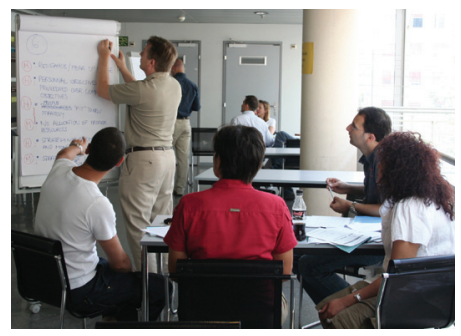

Fig. 1. Setting of the control condition

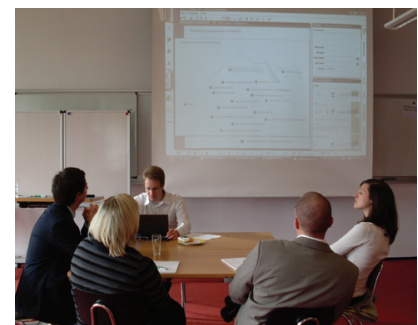

Fig. 2. Setting of the visualization-supported conditions

\subsection{Hypotheses}

Based on our previously stated research question, we propose five specific main hypotheses of the effect of visualization on group knowledge sharing:

In group knowledge sharing facilitation, an interactive, softwarebased visual support, compared to flipchart support, leads to greater:

H1. Productivity

a. Number of documented items

H2. Outcome quality

a. Range of documented items

b. Number of relevant items

c. Structure or categorization of items

d. Equal redundancies

H3. Learning

a. Recall

b. Knowledge gain

H4. Satisfaction

a. Satisfaction with process

b. Satisfaction with outcome

H5. Participation

a. Freedom of participation

b. Equality of participation

Our experimental study also aims to address how different kinds of visualization affect group collaboration. In order to evaluate this aspect, we have chosen two different visualizations, a seemingly fitting one (that other managers had rated as being helpful for knowledge sharing tasks) and a non-fitting (or unsuitable) one (that managers had indicated was not useful for supporting knowledge sharing tasks). Our hypothesis regarding these two differing graphic templates is: 
In group knowledge sharing facilitation, an optimal and a suboptimal visualization support will provide similar results (H1H5).

The rationale of this assumption is that we want to investigate if interactive visualization (that is, in general any kind of visualization template) can support knowledge sharing in teams. In order to do so, we have chosen to compare two very different visualization templates (as assessed by managers), to be used in the same software environment. If our hypothesis is confirmed (finding no difference between the two visualizations), then we are in a stronger position to generalize that visualization helps knowledge sharing, and that the findings are not specific to a particular visualization form (i.e. diagram, visual metaphor or knowledge map).

\subsection{Task and procedure}

As briefly stated before, the subjects had the task to share and document their knowledge in the group about strategy implementation problems and pitfalls [48]. This is a typical task in organizations in which small groups of managers have to share their prior knowledge and past experiences in order to avoid future problems. We have used let's focus [15], a software package developed by Reflact Inc. specifically for visualizing knowledge in a group setting. In this section, we outline the procedure followed in the experiment, as well as the user task given to the groups.

First, each subject was asked to write down (individually) a list of strategy implementation problems that he or she had already experienced directly or indirectly: participants were given five minutes for completing this task. This allowed us to measure the prior knowledge of each person participating in the experiment. We then randomly formed groups and randomly assigned them to one of the conditions. When the groups were formed, the participants selected a facilitator within their group; it should be noted that the participants were somewhat familiar with each other, because they attended the same executive MBA program. The facilitators of the two visualization conditions were instructed for 5 minutes on the main features of the visualization software and they had 5 more minutes to practice and familiarize themselves with the software environment. In particular they were instructed on how to map and edit contributions of their peers, insert icons, change colors, modify text and icons, resize, use freehand drawing and save their file. At the end of the training session the groups formed and they were given the same task instructions in written form (one page) that instructed them to discuss and document strategy implementation problems based on their experience, with the provided support (visualization software or flipchart). The facilitators in the visualization supported conditions had to capture the participants' contributions and place the text on the visual template provided. The facilitators were allowed to modify the templates slightly, but not to switch to another template. The groups had 45 minutes to complete this task. They were then asked to individually fill in a questionnaire in order for us to collect self-assessed measures of the meeting outcome as well as various control measures. After a one hour diversion task (a strategy lecture and an individual exercise), a recall test was administered to the subjects. We asked each participant individually to write down the strategy implementation problems discussed in his or her group that can be remembered. The participants were given 5 minutes for this recall task. Finally, we conducted a plenary debriefing session with all the participants. This allowed us to collect further qualitative information about the participants' perceptions, comparisons and problems.

\subsection{Selection of visualizations}

The selection of the visualization templates used for each condition is a critical choice for the reliability of the experimental results. In order to perform a rigorous selection, we have conducted a previous separate study to assess the suitability of different visualizations for the task of group knowledge sharing.

We developed a questionnaire for managers to quantitatively assess the fit of widely used visualizations in organizations with typical group activities. Twelve business visualization have been selected (Fig. 3), based on their popularity of use in organizations and based on their diversity of profile, evaluated with the Collaborative Dimensions of Visualization Framework [4]. The aim was to have a sample of widely used, but diverse visualizations. The survey respondents were all managers attending executive courses or seminars (and different from the experimental study subjects). For each visualization we asked survey participants to rate the fit or usefulness of each visualization (on a 5-point scale) for four main knowledge work tasks: idea generation, knowledge sharing, options evaluation and activity planning [14]. A paper with detailed results is under preparation.

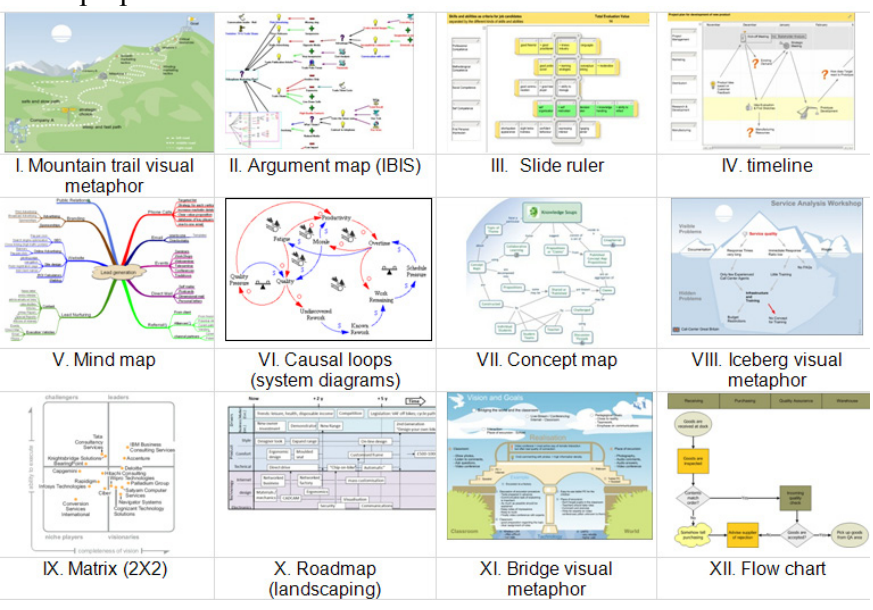

Fig. 3. The twelve visualizations evaluated in the template selection survey

The analysis of 116 completed questionnaires resulted in a ranking from the most to the least suitable visualization (in the managers' assessments) for each knowledge task. We do not assume that the participant's ratings are an objective assessment of each template, but they are an indication of the common perceptions of our target population (i.e., managers). We then used the manager's collective rankings of the visualizations, to select a particularly fitting (optimal) and non-fitting (suboptimal) visualization template for the knowledge sharing experiment.

The optimal visualization for the experiment resulted to be the iceberg visual metaphor (Fig. 4), which was chosen among the top rated visualizations for knowledge sharing.

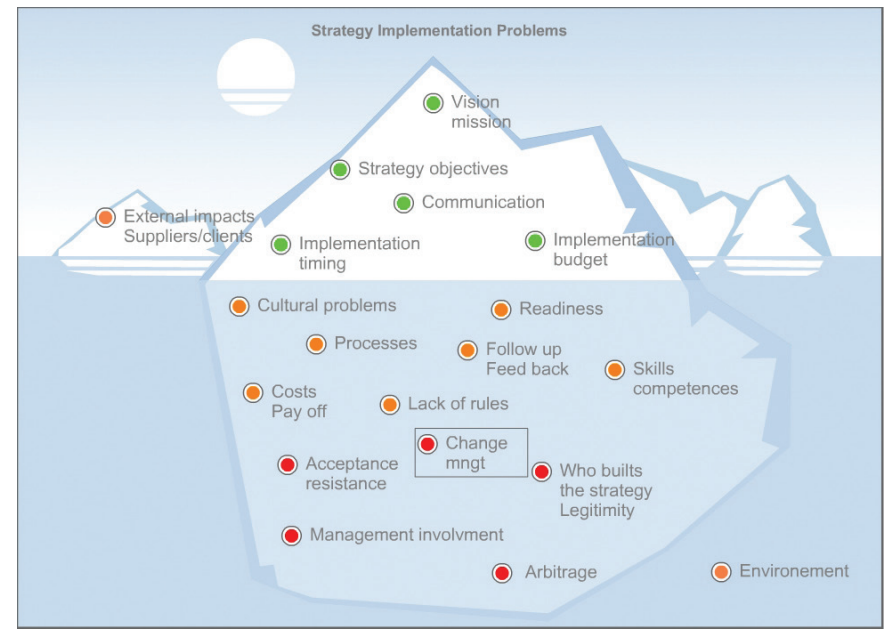

Fig. 4. Optimal visualization. Results of a group's collaboration. 


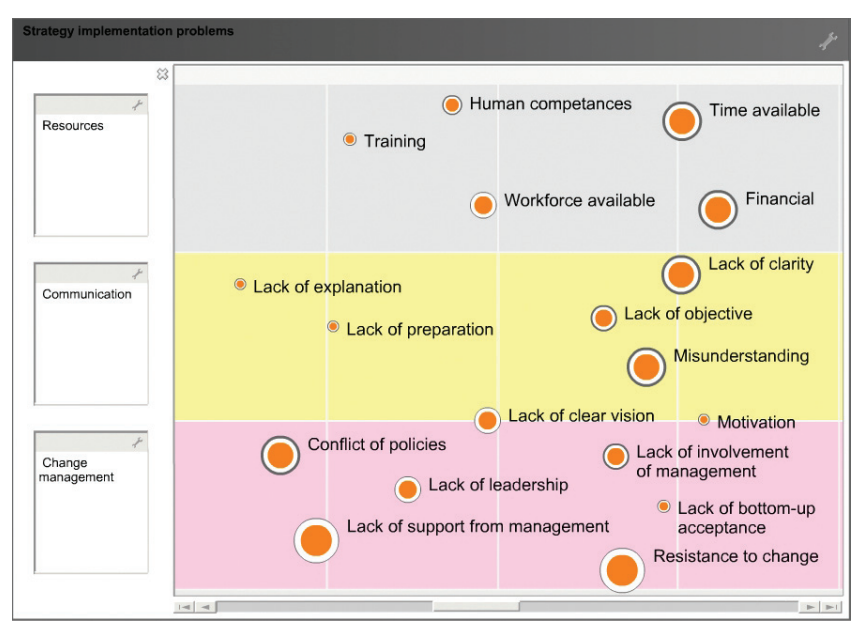

Fig. 5. Sub-optimal visualization. Results of a group's collaboration.

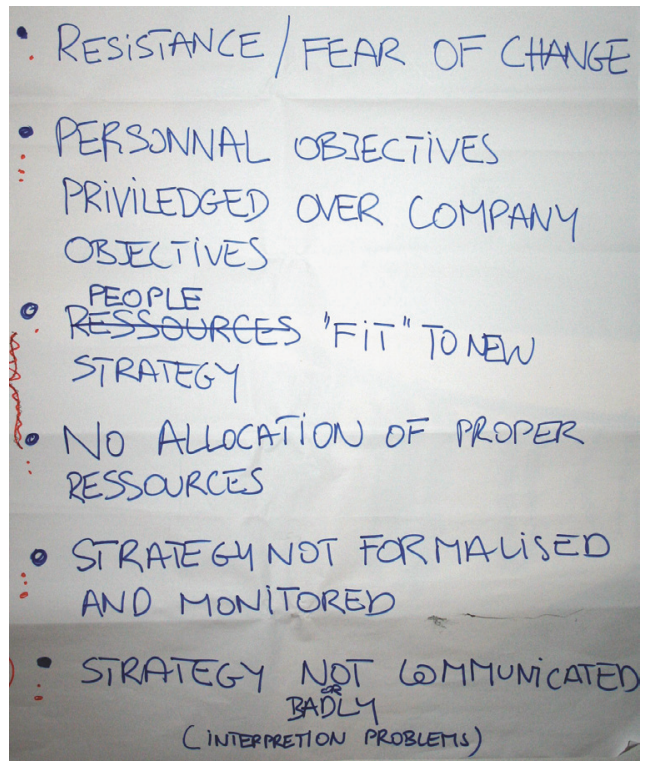

Fig. 6. Control condition. Results of a group's collaboration.

The suboptimal template was pulled from the lowest rated visualization (least fitting) regarding the knowledge sharing task, and resulted to be the timeline (Fig. 5).

The groups in the control condition were provided with plain flipchart paper and markers, to simulate the typical setting of a meeting in organizational contexts. We show the end result of a group in the control condition in Figure 6. It is important to highlight that the treatment groups were provided with empty visualization templates, thus without any text. In Figure 4 and Figure 5 we display the end results of two groups in the fitting and non-fitting (suboptimal) visualization condition respectively.

\subsection{Selection of subjects}

The subjects of this experiment were 131 managers attending a strategic management course (of which 101 were enrolled in an executive MBA program, and 35 were attending a three-day management seminar). Their average age was 36 , with an average of 11 years of work experience; 93 were men and 38 women. Their average familiarity with visualization supported meeting was 3.23 on a 7-point scale. Their familiarity with the topic of strategy implementation issues resulted to be 4.80 on average, rated on the same scale.

The groups were all composed of five people, except for one group which was composed of 6 . We have thus a total of 7 groups for the fitting visualization conditions, 7 groups for the non-fitting visualization condition, and 12 groups for the control condition (due to logistical reasons, it was not possible to perform more treatment conditions simultaneously). None of the subjects refused to take part in the experiment, but one group in the control condition had to be eliminated, because they had created an ad-hoc visualization on their flipchart, therefore potentially confounding our results. The sample characteristics are fairly distributed across interventions and we did not find significant differences between installments or countries (we conducted the experiment in three installments and in two different European countries).

Having managers as subjects, compared to undergraduate students, allows us to have less concerns regarding the external validity of the results, all else being equal. It also allows us to reduce potential concerns of future visualization users or managers regarding the experimental setting. We will discuss the limitations of our study in more details in section 5.3.

\subsection{Measurements}

We measured five main outcomes, based on the previously presented hypotheses: Productivity, Quality, Learning, Satisfaction and Participation. We assessed most of the outcomes with more than one evaluation variable. Table 1 summarizes each outcome with a brief description and the level on which it is measured, where $G$ stands for Group level measure, $I$ for Individual level measure and $O$ indicates that it is an Objective measure, while $S$ indicates a Subjective (selfreported) measure.

\section{Table 1. Outcomes description}

\begin{tabular}{|c|c|c|c|}
\hline H & Outcome & Description & Level \\
\hline $\begin{array}{l}1 \\
\mathrm{a}\end{array}$ & \multicolumn{2}{|l|}{ Productivity } & $\mathrm{G}, \mathrm{O}$ \\
\hline 2 & \multicolumn{2}{|l|}{ Quality of outcome } & $\mathrm{G}, \mathrm{O}$ \\
\hline $\mathrm{a}$ & Range of items & Diversity of items & \\
\hline b & Relevant items & $\begin{array}{l}\text { Number of relevant items as } \\
\text { identified in the literature [48] }\end{array}$ & \\
\hline $\mathrm{c}$ & Redundancies & Repetition of similar items & \\
\hline $\mathrm{d}$ & Structure & Number of category labels & \\
\hline 3 & \multicolumn{2}{|l|}{ Learning } & $\mathrm{I}, \mathrm{O}$ \\
\hline $\mathrm{a}$ & Recall & Total number of items recalled & \\
\hline $\mathrm{b}$ & Knowledge gain & $\begin{array}{l}\text { Correctly recalled items not } \\
\text { previously known }\end{array}$ & \\
\hline 4 & \multicolumn{2}{|l|}{ Satisfaction } & $\mathrm{I}, \mathrm{S}$ \\
\hline $\mathrm{a}$ & $\begin{array}{l}\text { Satisfaction with } \\
\text { process }\end{array}$ & Validated scales [5] & \\
\hline $\mathrm{b}$ & $\begin{array}{l}\text { Satisfaction with } \\
\text { outcome }\end{array}$ & Validated scales [5] & \\
\hline 5 & \multicolumn{2}{|l|}{ Participation } & $\mathrm{I}, \mathrm{S}$ \\
\hline $\mathrm{a}$ & $\begin{array}{l}\text { Participation } \\
\text { equality }\end{array}$ & Validated scale [49] & \\
\hline $\mathrm{b}$ & $\begin{array}{l}\text { Participation } \\
\text { freedom }\end{array}$ & $\begin{array}{l}\text { Ad-hoc scale (based on the } \\
\text { work of [29]) }\end{array}$ & \\
\hline
\end{tabular}

The quantity (Productivity) and quality of the group work [31] are measured at group level: Productivity is measured as the count of the documented items of strategy implementation problems by each group. Quality of the outcome is rated by two external coders, blind to the treatment and hypotheses, and assessed the Range of items (that is, the variety of the documented items), the Quantity of relevant items (how many of the six most relevant strategy implementation problems identified by Beer and Eisenstat [2]). The coders also assessed Redundancies as a control variable, to verify that a higher number of items is not due to repetitions. The coders identified similar items and counted these repeated ideas only once. We expect an equal number of redundancies per condition, independently of the total number of items identified, to assess that a higher Productivity is not due to the repetition of the same ideas. Structure identifies the number of categorizations or grouping levels that each group employed in carrying out the task (if any).Learning 
is measured at the individual level as the objective count of remembered items. Recall is a quantitative measure of the total number of items remembered in the recall test [28]. Knowledge gain measures the amount of "correct" items: that is, the number of items that are recalled from the group documentation, and not previously known (not present in the individual previous knowledge test). This measure is therefore more qualitative than the previous one, assessing the effective learning of discussed new items.

The last two measures of Satisfaction and Participation are selfreported by answering a questionnaire with 7 point scale answers (after the experiment). Satisfaction with Process and Satisfaction with Outcome are validated scales [5] of 4 items each, which have been employed in several previous studies. Equality of Participation is a 3 item scale developed and documented in [49]. We have developed a 4 item scale for Freedom of participation (partially based on the work of [29]) attempting to measure the degree to which subjects felt free and comfortable in participating in the group discussion and providing their contributions.

\section{RESULTS AND ANALYSIS}

We subsequently analyze the effect of the treatment on objective (Productivity, Quality, Learning) and subjective (Satisfaction, Participation) measures by performing an Analysis of Variance (ANOVA) on each metric. We report the means and standard deviation (in parenthesis) of the outcomes for each condition in Table 2.

Table 2. Mean comparison for treatment conditions

\begin{tabular}{|c|c|c|c|}
\hline 1.a Productivity & $\begin{array}{r}\text { Control } \\
13.58(5.25) \\
\end{array}$ & $\begin{array}{l}\text { Suboptimal } \\
18.29(3.35)\end{array}$ & $\begin{array}{r}\text { Optimal } \\
16.14(2.27) \\
\end{array}$ \\
\hline 2.a Range of items & $0.25(0.45)$ & $0.71(0.76)$ & $1.43(0.98)$ \\
\hline 2.b Relevant items & $5.08(1.00)$ & $6.71(2.56)$ & $5.57(1.81)$ \\
\hline 2.c Redundancies & $0.25(0.70)$ & $0.43(0.79)$ & $0.29(0.49)$ \\
\hline 2.d Structure levels & $0.25(0.45)$ & $1.29(0.49)$ & $1.57(0.53)$ \\
\hline 3.a Recall & $9.05(2.73)$ & $11.69(3.36)$ & $11.37(3.09)$ \\
\hline $\begin{array}{l}\text { 3.b Correct knowledge } \\
\text { gain }\end{array}$ & $5.8(2.25)$ & $8.52(2.90)$ & 8.4(3.09) \\
\hline $\begin{array}{l}\text { 4.a Satisfaction with } \\
\text { process }\end{array}$ & $5.85(0.84)$ & $5.47(0.93)$ & $5.88(0.64)$ \\
\hline $\begin{array}{l}\text { 4.b Satisfaction with } \\
\text { outcome }\end{array}$ & $5.82(0.77)$ & $5.66(0.95)$ & $5.98(0.67)$ \\
\hline $\begin{array}{l}\text { 5.a Equality of } \\
\text { participation }\end{array}$ & $5.59(1.11)$ & $5.36(1.04)$ & $5.72(0.96)$ \\
\hline $\begin{array}{l}\text { 5.b Freedom of } \\
\text { participation }\end{array}$ & $6.03(0.81)$ & $6.18(0.83)$ & $6.22(0.62)$ \\
\hline
\end{tabular}

Table 3 provides a summary of the ANOVA results (bold indicates significance at $95 \%$ confidence level, two tailed) which are discussed in more detail in the next sub-sections. We provide the significance test for the suboptimal condition compared to the control condition ("Suboptimal"), the optimal condition compared to the control condition ("Optimal"), the optimal condition compared to the suboptimal condition ("Optimal - Suboptimal") in order to test if the two visualizations are different with regard to their effects, and the two visualizations pooled (aggregated) together (optimal and suboptimal) compared to the control condition ("Visualizations Control").

\subsection{Group objective outcomes}

The ANOVA results show that the suboptimal (non-fitting) visualization leads to significantly higher Productivity (in terms of number of items elicited by the group). The optimal or fitting visualization also leads to higher productivity than the control condition, but the difference is not significant. Evaluating the two visualizations together, significant difference can be found from the control condition, while there is no statistical difference between the two visualizations.
Hypothesis 1a is not confirmed for the optimal or fitting visualization, yet it is confirmed for the Suboptimal or unfitting visualization. For Range of Items the optimal condition is significantly better than the control conditions, but the suboptimal condition is not. No significant difference can be found between the two visualizations, while the two visualization together result to be significantly better than the control condition. Hypothesis $2 \mathrm{a}$ is confirmed for the optimal visualization, for the aggregated visualizations, but not for the suboptimal visualization individually.

The outcome of Relevant items is significantly better for the suboptimal visualization compared to the control condition, while the optimal visualization is better, but the difference is not significant. There is no significant difference between the two visualizations, and the two visualizations together provide no difference from the unsupported condition. Hypothesis $3 \mathrm{~b}$ is confirmed only for the suboptimal visualization.

We found no significant difference for Redundancies between any of the conditions, as expected, therefore hypothesis $3 \mathrm{c}$ is confirmed for both visualizations. Structure levels is significantly better for both the optimal and the suboptimal condition, compared to the control condition, and there is no significant difference between the two visualizations. Hypothesis $4 \mathrm{~d}$ is confirmed for both visualizations.

\subsection{Individual objective outcomes}

Recall is significantly better when the optimal or the suboptimal visualizations are used, compared to the control condition. No significant difference is found between the two visualizations. Hypothesis $3 \mathrm{a}$ is confirmed in full.

Knowledge gain leads to the same results, with the two visualization being significantly different from the control condition, and therefore confirming hypothesis $3 b$.

\subsection{Individual subjective outcomes}

The individual subjective outcomes of Satisfaction and Participation have been measured through a questionnaire, that the participants had been asked to fill-in at the end of the treatment. As a first step, we have assessed the scales by conducting a principal component analysis on the four scales together and the results confirm the four desired components for each scale with loadings well above 0.7 for most of the items. More detailed results are available upon request.

Secondly we have tested the reliability of each scale, which resulted in a Cronbach's Alpha of 0.905 for Satisfaction with process (4 item validated scale), 0.901 for Satisfaction with outcome (4 item validated scale), 0.920 for Equality of Participation (3 item validated scale) and 0.870 for Freedom of Participation (4 item ad hoc scale). This scale was employed for the first time and is performing well in terms both of principal component analysis factor loadings and of reliability. Since all the scales result to work properly, we proceed with the Analysis of Variance.

As can be easily inferred from the results in Table 3, no statistical difference can be found between the treatments and any of the subjective measures. Hypotheses $4 \mathrm{a}, 4 \mathrm{~b}, 5 \mathrm{a}$ and $5 \mathrm{~b}$ are therefore not confirmed.

\subsection{Covariates and interactions}

We have tested the effect of potential confounding variables by adding them to the treatment model. However, we find no relevant statistical difference in the significance levels of the treatment effect when accounting for the control measures (i.e. facilitator role, prior knowledge, gender, etc.). We tested the interaction of treatment and relevant covariates (ANCOVA), to test the hypothesis that there is treatment heterogeneity per condition, and we did not find statistical support for that problem. We have also tested the residuals and we find normality in their distribution. 
This implies that the above presented findings on treatment effect are strong and stable regardless of context, demographic and experience differences. Detailed results are available upon request.

Table 3. ANOVA results

\begin{tabular}{ll|ll}
\hline Outcome & $\mathbf{p}$ & Outcome & $\mathbf{p}$ \\
\hline la. Productivity & & 3a. Recall & \\
Suboptimal & $\mathbf{. 0 2 7}$ & Suboptimal & $\mathbf{. 0 1 3}$ \\
Optimal & .210 & Optimal & $\mathbf{. 0 4 9}$ \\
Optimal - Suboptimal & .347 & Optimal - Suboptimal & .584 \\
Visualizations - Control & $\mathbf{. 0 3 7}$ & Visualizations - Control & $\mathbf{. 0 0 8}$ \\
\hline & & 3b. Correct knowledge & \\
2a. Range of items & & gain & \\
Suboptimal & .179 & Suboptimal & $\mathbf{. 0 0 2}$ \\
Optimal & $\mathbf{. 0 0 2}$ & Optimal & $\mathbf{. 0 1 0}$ \\
Optimal - Suboptimal & .070 & Optimal - Suboptimal & .558 \\
Visualizations - Control & $\mathbf{. 0 0 7}$ & Visualizations - Control & $\mathbf{. 0 0 1}$ \\
\hline & & 4a. Satisfaction with & \\
2b. Relevant items & & process & \\
Suboptimal & $\mathbf{. 0 4 2}$ & Suboptimal & .138 \\
Optimal & .562 & Optimal & .905 \\
Optimal - Suboptimal & .233 & Optimal - Suboptimal & .154 \\
Visualizations - Control & .136 & Visualizations - Control & .401 \\
\hline & & 4b. Satisfaction with & \\
2c. Redundancies & & outcome & \\
Suboptimal & .514 & Suboptimal & .467 \\
Optimal & .896 & Optimal & .498 \\
Optimal - Suboptimal & .642 & Optimal - Suboptimal & .217 \\
Visualizations - Control & .635 & Visualizations - Control & .976 \\
\hline & & 5a. Equality of & \\
2d. Structure & & participation & .474 \\
Suboptimal & $\mathbf{. 0 0 0}$ & Suboptimal & .721 \\
Optimal & $\mathbf{. 0 0 0}$ & Optimal & .343 \\
Optimal - Suboptimal & .281 & Optimal - Suboptimal \\
Visualizations - Control & $\mathbf{. 0 0 0}$ & Visualizations - Control & .832 \\
\hline & & 5b. Freedom of & \\
& & participation & Suboptimal \\
\hline
\end{tabular}

\section{DIscussion}

\section{$5.1 \quad$ Findings}

The objective of this research was to shed light on the role of visualization in supporting collaborative knowledge sharing in groups. The empirical results presented in the above section lead to four main findings.

First, a clear pattern in the significance levels can be noticed. For all the objective measures (at group and at individual level) the use of interactive visualization results in a significant improvement of performance compared to the control condition, with the individual measures offering stronger results than the group level measures. On the contrary, visualization has no effect on any of the subjective measures, compared to the control condition. The implication of these results is that visualization leads to objectively better results, but subjects do not perceive this difference. We believe these finding to be highly revealing of the hidden beneficial effects of visualization in collaborative settings. By pulling together subjective and objective measures, we can compare the real to the perceived gain of using visualization for supporting group collaboration. Future research could focus on exploring the reasons for this discrepancy between objective and subjective results. We can also hypothesize that subjects do not have a reference or comparison level when completing in the questionnaire (since they are not provided with feedback or comparisons with other groups' results at that point in time), therefore they cannot assess their satisfaction and group participation in relative terms.

Second, we find that for none of the outcomes there is a significant difference between the two visualization conditions, confirming our hypothesis that the optimal/fitting and the suboptimal/non-fitting visualization will lead to similar results. This finding suggests that although visualization templates for knowledge sharing can differ in their shape or form, they do not necessarily have to lead to significant differences in team performance with regard to group knowledge sharing.

The third implication of the experiment's results is that the results are very consistent across contexts, as we found no difference between installments, and no impact of the covariates on the treatment results. This implies that the effect of visualization is constant regardless of context and therefore can be predicted.

Finally we can point out that the interactive visualization support in the groups has not lead to a single inferior result when compared to the flip chart groups. Some of the outcomes were positively affected by the use of visualization software, while some were not affected, but none of the outcomes have been affected negatively by the use of visualization for facilitating group knowledge sharing.

\subsection{Implications and Recommendations}

Our findings have implications for both information visualization and for organization studies. The results show that using softwarebased, interactive visualization for supporting group knowledge sharing can be especially helpful to advance the individual learning and recall for the group participants. We also found that this kind of real-time visualization increases the quality and quantity of the shared experiences. Consequently, using a flexible visual template when facilitating a group knowledge sharing meeting, can lead to several significant advantages (and does not seem to cause major negative effects). By contrast, we have found that the participants in the visualization supported teams are not more satisfied than the textoriented flip chart groups with regard to their results or collaboration process. They also do not perceive a greater level of participation than their control group peers. Similar findings are common in GSS studies [8, 27]. In the information visualization domain, Andrews [1] finds that "Users will apparently need a great deal of persuading to move from a familiar trusted interface to a new, unfamiliar one." This might be the case also in our study, where subjects are not familiar with the employed software.

Our study found no statistically significant difference between the group outcomes of the two visualizations. This has implications for the information visualization community, as it seems to show that interactive visualization is helpful, regardless of its specific form (visual metaphor, knowledge map or diagram).

Indeed, research in the field of memory, has for some time indicated that images can enhance recall and learning better than verbal descriptions [12]. We can observe that the two employed visualizations do not lead to different results, but that they are significantly better than the control condition regarding productivity and quality of outcome. Henderson provides an explanation for this phenomenon: "Why are visual representations so powerful? I have suggested that it is their meta-indexical quality - their ability to be a holding ground and negotiation space for both explicit and yet-to-bemade-explicit knowledge- that allows them to be more than the sum of their parts. [...] One very important capacity of the visual lies in its malleability - its ability to be drawn interactively and shaped and redrawn and reshaped [...]. In this process, the visual representation integrates and informs the collective and changing cognition of those designing it" [20].

It is interesting to note that most of the groups assigned with a sub-optimal visualization did not use the visualization "appropriately". They were assigned the timeline template, but six of 
the seven groups in this condition ignored the time axis (as can be seen in Fig. 5). Only one of the groups used both axes, adapting the y axis of time by using only two time frames, which they named "planning" and "implementation". We can assume that the suboptimal visualization has been re-appropriated by the groups and adapted to their discussion needs. Conversely, only one of the seven groups assigned with the optimal visualization (the iceberg visual metaphor) used it in a different way than intended. This appropriation process has been the focus of the Adaptive Structuration Theory (AST), which highlights the role of group interaction in appropriating structures to guide further interactions. Consequently groups may use technology "in ways intended by the system designers, or they may use it in opposing ways"; unintended appropriation has indeed been observed in other experimental studies [13]. The re-appropriation effect could also be the object of further study in the information visualization field, to investigate when mapping functionalities are used in different ways than intended by the developers. This is also in line with Shneiderman's and Plaisant's call to action: "We seek to encourage information visualization researchers to study users doing their own work in the process of achieving their goals." [38].

In the control condition, all the considered groups documented the elicited items in a list. They often deleted or corrected words or sentences by drawing a straight or wavy line on them (as in Fig. 6 for the word "resources"). Only one of the twelve groups used a categorization (on three levels). Most of the groups, after finishing their discussion, wrote all the items on a new flipchart paper for presentation to the class, in a more legible form.

\subsection{Limitations and future research}

One of the strengths of the study reported in this paper is, we believe, that the subjects of the experiment are real managers with several years of work experience and are familiar with one another, thus representing the target population of the study (managers) in a quasirealistic setting. This is a stark contrast to other studies in this domain that mostly use undergraduate students in their user experiments. A limitation ensuing from this choice of subjects regards the scope of the questionnaire issued to participants that had to remain concise. Other limitations introduced by the experimental setting include possible personal preferences on behalf of the subjects for the use of visualization in general or for a specific visualization template. Subjects of the experiment are managers with high education levels (typically university graduates) and the generalization of the experiment results might be limited to educated middle managers in organizations from Western Europe. Another limitation of this study is that we do not account for the effect of visualization in a non-interactive (or non electronic) context, such as on brown papers, whiteboards, posters, or flipcharts, therefore we are not able to isolate the effect of visualization from the effect of computer interaction: our research group is currently working on comparing paper based and interactive visualizations. We also acknowledge the limited scope of the visualizations used in our experiment, as each group facilitator was asked to place the participant's contributions as moveable text items on a graphic template. Furthermore, we had only one person that could use the mouse and keyboard (the facilitator), in order to simulate a typical group meeting in a corporate setting. Future research could study the effect of multiple and simultaneous user interactions (for example with multi-mice support or with a multi touch screen / wall).

The employed research design can still lead to further investigations of several variations, by replicating the experiment in different settings or with varying conditions, and therefore measuring their differential impact on the outcomes that we have found. The same experiment could be replicated for varying group sizes or for different knowledge tasks, such as assessment, idea generation or planning, or in different cultural context (such as in Asia) [25] [42].
In order to increase the external validity of our findings, the same measures could be replicated in the field, in an actual organization, through a situated [18] or field [19] experiment.

\section{Conclusion}

The results from this experimental study add to the growing literature on collaborative information visualization. In particular we have focused on analyzing co-located and synchronous collaboration through visualization for a group knowledge sharing task. Our main research hypothesis was that a visualization-supported meeting leads to better performance than a non-supported condition.

In this paper, we have first contextualized our research and argued for the relevance of our research question (atypical in the IV community). We have then described the research design, procedure and outcome measures in order to assess the effects of two different visualizations (optimal and suboptimal) compared to an unsupported control condition in group knowledge sharing. Next we have presented the results of the experiment that clearly show that using interactive visualization for facilitating collaborative knowledge sharing increases individual learning and team performance (both in terms of quality and quantity). Internal validity is ensured by the rigorous experimental setting with random attribution of subjects to groups and conditions. In addition, the choice of managers as subjects, and of a typical organizational topic as the base of the discussion (strategy implementation problems), allows to have a reasonable degree of external validity.

Our work aims to be a contribution to the understanding of the role of collaborative information visualization in a non-numeric context, that is to say where the information that is visualized is not based on existing (quantitative) data sets, but rather on manager's prior experiences and insights (although the idea of real-time group annotations and interpretations can also be applied to quantitative information visualization, see below). We consider that examining this topic is highly relevant, both at a theoretical and at a practical level to move the information visualization field further and expand its scope. The novelty of our research is to be found in applying the theories and findings of the collaborative information visualization field to the specific context and needs of organizational group meetings, and demonstrating that visualizing conversations interactively improves learning and group performance.

We believe this study to be relevant for the information visualization community, as it provides evidence that the use of interactive visualization, compared to an unsupported setting, leads to statistically significant better performances, in the context of knowledge sharing in organizations.

One implication of our study for information visualization scholars and developers may thus be the suggestion to include collaborative annotation functionalities to their data mapping tools in order to help in the collective sense making [42] of the provided data visualizations. As our study shows, such positioned annotations can lead to numerous positive effects for group deliberation.

\section{ACKNOWLEDGMENTS}

The authors wish to thank the four anonymous reviewers of the INFOVIS09 conference for their helpful suggestions. We are also grateful to the participants of the IKNOW'08 conference (Graz, 2008) and of the Scent of Information Workshop (Linz, 2009) for their valuable feedback on this research.

\section{REFERENCES}

[1] K. Andrews. Evaluating Information Visualisations. In AVI 2006 Workshop BELIV'06, Venice, Italy, pages 1-5, 2006.

[2] M. Beer and A. R. Eisenstat. The Silent Killers of Strategy Implementation and Learning. Sloan Management Review, 41: 29-40, Summer 2000, 2000. 
[3] M. Bitter-Rijpkema, R. Martens, and W. Jochems. Supporting knowledge elicitation for learning in virtual teams. Educational Technology \& Society, 5: 113-118, 2002.

[4] S. Bresciani, A. F. Blackwell, and M. Eppler. A Collaborative Dimensions Framework: Understanding the Mediating Role of Conceptual Visualizations in Collaborative Knowledge Work. In HICCS 2008, Hawaii, pages 180-189, 2008.

[5] R. O. Briggs, B. A. Reinig, and G. de Vreede. Meeting Satisfaction for Technology-Supported Groups: an Empirical Validation of a GoalAttainment Model. Small Group Research, 37: 585-611,2006.

[6] D. T. Campbell and C. J. Stanley. Experimental and quasi-experimental design for research. Chicago, USA: Rand McNally College Pub. Co., 1966.

[7] S. K. Card, J. Mackinlay, and B. Shneiderman. Readings in Information Visualization: Using Vision to Think. San Francisco, CA: Morgan Kaufmann, ed., 1999.

[8] J. M. Carey and C. J. Kacmar. The impact of communication mode and task complexity on small group performance and member satisfaction. Computers in Human Behavior, 13: 23-49, 1997.

[9] P. R. Carlile. A Pragmatic View of Knowledge and Boundaries: Boundary Objects in New Product Development. Organization Science, 13: 442-455, Jul/Aug 2002, 2002.

[10]C. Chen and Y. Yu. Empirical studies of information visualization: a meta-analysis. International Journal of Human-Computer Studies, 53: 851-866, 2000.

[11] M. C. Chuah and S. F. Roth. Visualizing Common Ground. In Seventh International Conference on Information Visualization (IV'03), London, U.K., pages 365- 372, 2003.

[12] J. M. Clark and A. Paivio. Dual coding theory and education. Educational Psychology Review, 37: 250-263,1991.

[13] G. DeSanctis and M. S. Poole. Use of group decision support systems as an appropriation process. In Proceedings of the Twenty-Second Annual Hawaii International Conference on System Sciences, Kailua-Kona, HI, USA, pages 149-157, 1989.

[14] M. Eppler and J. Mengis. Das Management von Wissensdialogen Zeitschrift für Organisationsentwicklung, 4/05: 14-23,2005.

[15]M. J. Eppler. Facilitating Knowledge Communication through Joint Interactive Visualization. In I-KNOW'04, Graz, Austria, 2004.

[16] M. J. Eppler and K. Platts. Visual Strategizing: The Systematic Use of Visualization in the Strategic Planning Process. Long Range Planning LRP - International Journal of Strategic Management, 42: 42-74, February 2009, 2009.

[17] J. Fjermestad and S. R. Hiltz. An assessment of group support systems experiment research: methodology and results. Journal of Management Information Systems, 15: 143, 1998.

[18] J. Greenberg and E. C. Tomlinson. Situated Experiments in Organizations: Transplanting the Lab to the Field. Journal of Management, 30: 703-724,2004.

[19] G. W. Harrison and J. A. List. Field Experiments. Journal of Economic Literature, 42: 1009-1055, 2004.

[20]K. Henderson. On Line and On Paper: Visual Representations, Visual Culture, and Computer Graphics in Design Engineering Baskerville, U.S.A.: The MIT Press, 1998

[21] P. Isenberg and S. Carpendale. Interactive Tree Comparison for Colocated Collaborative Information Visualization. IEEE Transactions on Visualization and Computer Graphics, 13: 1232-1239, November/ December, 2007.

[22] J. H. Larkin and H. Simon. Why a Diagram is (Sometimes) Worth Ten Thousand Words. Cognitive Science, 11: 65-99, 1987.

[23] G. Mark, K. Carpenter, and A. Kobsa. A Model of Synchronous Collaborative Information Visualization. In Seventh International Conference on Information Visualization (IV'03), London, U.K. , pages 373-381, 2003.

[24] G. Mark, A. Kobsa, and V. Gonzalez. Do Four Eyes See Better than Two? Collaborative versus Individual Discovery in Data Visualization Systems. In Sixth International Conference on Information Visualisation (IV'02), pages 249 - 255, 2002.

[25] J. E. McGrath. Groups: interaction and performance Englewood Cliffs, NJ: Prentice-Hall, 1984.

[26] R. J. Mejias, M. M. Shepherd, D. R. Vogel, and L. Lazaneo. Consensus and perceived satisfaction levels: A cross-cultural comparison of GSS and non-GSS outcomes within and between the United Stated and Mexico. Journal of Management Information Systems 13: 137-161, Winter 1996/1997, 1996.
[27] J. Mengis. Integrating Knowledge through Communication: An Analysis of Expert-Decision Maker Interactions. In Institute of Corporate Communication. PhD Lugano, Switzerland: University of Lugano (USI): 460, 2007.

[28] A. M. O’Donnell, D. F. Dansereau, and R. H. Hall. Knowledge Maps as Scaffolds for Cognitive Processing. Educational Psychology Review, 14: 71-86, March 2002, 2002.

[29] S. Paul, P. Seetharamanb, I. Samarah, and P. P. Mykytyn. Impact of heterogeneity and collaborative conflict management style on the performance of synchronous global virtual teams. Information \& Management, 41: 303-321, January 2004, 2004.

[30]R. Phaal and G. Muller. Towards Visual Strategy: An Architectural Framework for Roadmapping. In PICMET 2007, Portland, Oregon USA, 2007.

[31] B. A. Reinig, R. O. Briggs, and J. F. J. Nunameker. On the measurement of Idea Quality. Journal of Management Information Systems, 23: 143161,2007

[32] G. Robertson, R. Fernandez, D. Fisher, B. Lee, and J. Stasko. Effectiveness of Animation in Trend Visualization. IEEE Transactions on Visualization and Computer Graphics, 14: 1325-1332, November/December 2008, 2008.

[33] A. C. Robinson. Collaborative Synthesis of Visual Analytic Results. In Visual Analytics Science and Technology, VAST '08. IEEE Symposium on, Columbus, OH, pages 67-74, 2008.

[34] Y. Rogers, H. Brignull, and M. Scaife. Designing Dynamic Interactive Visualisations to Support Collaboration and Cognition. In Sixth International Conference on Information Visualisation (IV'02), London, U.K., pages 39 - 48, 2002.

[35] J. Roos, V. Bart, and M. Statler. Playing Seriously with Strategy Long Range Planning, 37: 549- 568,2004.

[36] B. Shneiderman. Computer Science: Science 2.0. Science, 319: 1349$1350,2008$.

[37] B. Shneiderman. The Eyes Have It: A Task by Data Type Taxonomy for Information Visualizations. In IEEE Visual Languages, Boulder, CO, pages 336-343, 1996.

[38] B. Shneiderman and C. Plaisant. Strategies for Evaluating Information Visualization Tools: Multi-dimensional In-depth Long-term Case Studies. In AVI 2006 Workshop BELIV'06, Venice, Italy, 2006.

[39] S. L. Star and J. R. Griesemer. Institutional Ecology, 'Translations' and Boundary Objects: Amateurs and Professionals in Berkeley's Museum of Vertebrate Zoology, 1907-39. Social Studies of Science, 19: 387-420, 1989

[40]D. D. Suthers. Collaborative Knowledge Construction through Shared Representations. In 38th Hawaii International Conference on System Sciences (HICSS), Big Island, HI, USA, 2005.

[41] B. C. Y. Tan, K.-K. Wei, R. T. Watson, and R. Walczuch. Reducing status effects with computer-mediated communication: Evidence from two Distinct National Cultures. Journal of Management Information Systems, 15: 119-141, June 1998, 1998.

[42] J. J. Thomas and K. A. Cook. Illuminating the path: the research and development agenda for visual analytics. Los Alamitos, CA: Institute of Electrical and Electronics Engineers; National Visualization and Analytics Center; United States. Dept. of Homeland Security. IEEE Computer Society, 2005

[43] E. R. Tufte. Envisioning Information. Cheshire, Connecticut: Graphic Press, 1990.

[44]E. R. Tufte. Visual Explanations. Images and Quantities, Evidence and Narrative. Cheshire, Connecticut: Graphic Press, 1997.

[45] B. Tversky. Visuospatial reasoning. In Handbook of Reasoning, K. Holyoak and R. Morrison, Eds. Cambridge, UK: Cambridge University Press, pages 209-249, 2005.

[46] C. Ware. Information Visualization (2nd Edition). San Francisco CA: Morgan Kaufmann, 2004.

[47] Z. Wen and M. X. Zhou. Evaluating the Use of Data Transformation for Information Visualization. IEEE Transaction on Visualization and Computer Graphics, 14: 1309-1316, November/December 2008, 2008.

[48] L. Yang, G. Sun, and M. Eppler. Making Strategy Work: A Literature Review on the Factors influencing Strategy Implementation. In ICA Working Paper \#2/2008 Lugano, Switzerland: University of Lugano (USI): 46, 2008.

[49] R. W. Zmud, R. J. Mejias, B. A. Reinig, and I. M. Martinez-Martinez. Participation equality: Measurement within collaborative electronic environments: a three country study. In Purdue CIBER working papers, 2002 . 